\title{
Older adults under a mixed regime of infectious and chronic diseases
}

\author{
Rafael Samper-Ternent, MD, (1) Alejandra Michaels-Obregon, MSc, MBA, ${ }^{(2)}$ \\ Rebeca Wong, PhD, ${ }^{(2)}$ Alberto Palloni, PhD. ${ }^{(3)}$
}

Samper-Ternent R, Michaels-Obregon A, Wong R, Palloni A. Older adults under a mixed regime of infectious and chronic diseases. Salud Publica Mex 20I2;54:487-495.

\begin{abstract}
Objective. Analyze the impact of a mixed regime of infectious and chronic conditions among older adults in Mexico on their health progression. Materials and methods. A total of 12207 adults from the Mexican Health and Aging Study were included. Logistic regression analyses were conducted to assess the relationship between self-reported health (SRH) and covariates, including infectious and chronic diseases. Changes in SRH between 200 I-2003 were analyzed using multinomial analysis. Results. Older age, low SES, poor SRH and type of disease at baseline increase the odds of poor SRH at follow-up. Odds of poor SRH are highest for persons with both types of diseases (OR 2.63, SE 0.24), followed by only chronic (OR I.86; SE 0.12) and finally only infectious (OR I.55; SE 0.25). Conclusion. Mexico is experiencing a mixed regime of diseases that affects the health and wellbeing of older adults. Despite the rising importance of chronic diseases in countries like Mexico, it is premature to disregard the relevance of infectious diseases for public health.
\end{abstract}

Key words: older adults; mixed epidemiological regime; infectious diseases; chronic diseases; Mexico
Samper-Ternent R, Michaels-Obregon A, Wong R, Palloni A. Adultos mayores en un régimen epidemiológico mixto de enfermedades infecciosas y crónicas. Salud Publica Mex 20I2;54:487-495.

\section{Resumen}

Objetivo. Analizar el impacto del régimen epidemiológico mixto presente en México sobre la salud de los adultos mayores. Material y métodos. Participaron adultos mayores de la Encuesta Nacional de Salud y Envejecimiento en México $(n=12$ 207). Se usaron regresiones logísticas para evaluar la relación entre autorreporte de salud (ARS) y variables como enfermedades infecciosas y crónicas. Se analizó el cambio en ARS mediante análisis multinomiales. Resultados. Edad, estatus socioeconómico, pobre ARS y tipo de enfermedad aumentan el riesgo de pobre ARS en el futuro. La razón de probabilidades de pobre ARS fue más alta para individuos con ambos tipos de enfermedad (OR 2.63, SE 0.24), seguida por aquellos con crónicas (OR I.86; SE 0.12) y aquellos con infecciosas (OR I.55; SE 0.25). Conclusión. El régimen mixto presente en México afecta a los adultos mayores. En países como México, es prematuro dejar de lado el impacto de las enfermedades infecciosas para enfocarse únicamente en prevenir las crónicas.

Palabras clave: adultos mayores; régimen epidemiológico mixto; enfermedades infecciosas; enfermedades crónicas; México

(I) Sealy Center on Aging, University of Texas Medical Branch. Galveston, TX, USA.

(2) WHO/PAHO Center on Aging and Health, University of Texas Medical Branch. Galveston, TX, USA.

(3) Center for Demography and Ecology, University of Wisconsin. Madison, WI, USA.

Received on: June 22, 20II Accepted on: May 24, 2012

Corresponding author: Rafael Samper-Ternent, MD. Sealy Center on Aging, University of Texas Medical Branch. Rebeca Sealy Room 5.I02, 30I University Boulevard. Galveston, TX 77555-0I77 USA.

E-mail: rasamper@utmb.edu 
A well-known account of health and mortality changes originally proposed by Abdel Omran states that populations undergo three stages of the so-called epidemiological transition. ${ }^{1}$ This framework has been interpreted in a number of ways but in all cases the original structure remains unaltered..$^{2-4}$ First, there is a presumption that societies move from initial regimes ruled by injuries and infectious diseases to a terminal one dominated by chronic conditions, particularly cancer and cardiovascular disease. Second, although Omran's original model doesn't maintain that all populations experience the same stages in a particular sequence or following a particular timing, users of the framework frequently presume that historical transitions must somehow be ordered. ${ }^{2,3,5}$ Third, both the original formulation and its many applications seem to ignore the underlying forces that trigger changes within or between the stages.

Omran's framework is quite useful to describe changes experienced by high-income countries, however, its applicability to low-income countries is questionable. Most low-income countries, particularly those in Latin America, left behind regimes dominated by infectious diseases and are experiencing high death rates associated with cancer, cardiovascular disease and metabolic disorders. ${ }^{6}$ Meanwhile, conventional (and emerging) infectious diseases remain highly prevalent, to the point that mortality attributable to infectious diseases is higher than would be expected given the mortality rates associated with chronic diseases. ${ }^{7}$ The mixed regime of infectious and chronic diseases has theoretical implications for health status, mortality and wellbeing of susceptible populations, in particular older adults.

Populations in Latin America attaining older ages after the year 2000 will be particularly fragile due to unfavorable childhood experiences. ${ }^{7}$ These countries experienced rapid mortality decline beginning in 1930 or 1940 because of increased medical knowledge and technology and to a lesser degree, by improvements in living standards. This, in addition to life-style exposures and changes, produces three outcomes. First, a decrease in infectious diseases and a corresponding rise in chronic conditions. Second, increasing frailty of cohorts born right before, during and shortly after the interventions. And third, a persistence of infectious diseases since some of their root causes still remain. Mexico is an example where chronic diseases are increasing similar to some developed countries and yet, infectious diseases are still common and costly causes of hospital and ambulatory care. ${ }^{8}$ Whether or not and how this mixed regime of diseases affects the wellbeing of older Mexican adults is not well understood.

The current study will use Mexico to highlight likely consequences of a mixed regime of diseases for older adults. Since infectious and chronic diseases elicit different biologic responses we seek to understand the health and mortality consequences of the co-existence of infectious and chronic diseases. We will determine how self-reported health (SRH) changes in the presence of different disease categories after controlling for socioeconomic status (SES). It is well known that self-reported health status is an important predictor of mortality and is strongly associated with subjective wellbeing., ${ }^{9,10}$ Thus, identification of the effects of mixed health regimes on SRH has potential implications for population health.

\section{Materials and methods}

\section{Analysis sample}

Data for this study comes from the Mexican Health and Aging Study (MHAS, or its name in Spanish ENASEM, Estudio Nacional de Salud y Envejecimiento en México). The MHAS is a prospective panel study, nationally representative of rural and urban areas. This study contains information for adults 50 years and older that resided in Mexico in 2001, as well as their spouse or partner. The MHAS was designed to examine the aging process and evaluate the impact of disease on health, function, and mortality. There are two waves of information available so far: baseline, collected in 2001 and follow-up, collected in 2003. A more detailed description of the study is available elsewhere. ${ }^{11,12}$ This paper uses data from both waves of the study. The analysis sample includes adults 50 years and older with complete information on all relevant variables at baseline $(n=11379)$ and followup $(\mathrm{n}=11$ 194).

\section{Variables}

SRH is the dependent variable for our models and was measured by asking respondents to rate their health with a 5-item scale ranging from poor to excellent. The variable has a skewed distribution so we dichotomized it as poor vs. non-poor health to determine how type of disease relates to SRH. "Poor" was one category and fair, good, very good and excellent were grouped as "nonpoor". Previous literature has demonstrated that poor SRH increases mortality risk. ${ }^{13,14}$ Additionally, changes in SRH predict changes in mortality risk. ${ }^{15}$

We also used age (continuous), ${ }^{*}$ gender, marital status, residence (urban/rural), assets, wealth index,

\footnotetext{
* In separate analysis we included age squared in the regression to test for nonlinearity. However, the goodness of fit did not improve and the estimator was not significant.
}

salud pública de méxico / vol. 54, no. 5, septiembre-octubre de 2012 
and education reported at baseline (2001) as covariables. Marital status was categorized as: unmarried (single, divorced or separated), married (in a marriage or cohabitation), and widowed. Residence was dichotomized as rural and urban using 100000 inhabitants as the cut-off point. Assets were included at the individual level as a continuous variable. Data for assets was collected in Mexican pesos, valued at the time of interview; using the total value of assets minus debts. ${ }^{16} \mathrm{~A}$ wealth index was included in the analysis because it is a more accurate and equitably distributed indicator of economic status than income. ${ }^{17,18}$ We used principal component analysis (PCA) to construct this variable including four dwelling characteristics and six durable goods for the index. Others have reported reliability and usefulness of results from PCA analysis including dwelling characteristics and consumer durables to capture economic status among older adults. ${ }^{19}$ The Cronbach's alpha (0.86) indicated a high internal reliability of the wealth index with the variables included. Only the first factor from the PCA was used because it explained $47.4 \%$ of the variance with an eigen value of 4.3. The wealth index was included in the models coded as low, medium and high. Due to low educational attainment in our sample, we categorized education as: low ( $<3$ years), medium (3-5 years), and high ( $>6$ years).

Self-report of four infectious diseases was used: liver or kidney infection, tuberculosis and pneumonia. Respondents were asked "In the last two years, has a doctor or medical personnel told you that you have...?" for each disease. * Self-report of seven chronic diseases was assessed: hypertension, diabetes, heart attack, cancer, lung disease, stroke, and arthritis. Respondents were asked "Has a doctor or medical personnel ever told you that you have...?" for each disease. Disease types were dichotomized (yes vs. no) and grouped to form four categories of at least one reported disease: infectious, chronic, both (infectious and chronic) and neither. Death could not be used as a separate transition group because of small sample size, for example, only 14 individuals had died between both waves in the infectious disease category. Thus death was included with poor SRH at follow-up.

\section{Statistical analysis}

Descriptive statistics are reported as means and standard deviations for continuous variables and percentages for categorical variables initially presented by

\footnotetext{
* The follow-up question was modified to assess incidence of infectious or chronic diseases between waves.
}

four disease categories (infectious, chronic, both and neither) at baseline. Sampling weights are used for these analyses. To describe the change between waves, data is presented by SRH (poor vs. non-poor) in 2003 according to 2001 characteristics.

To identify the risk of poor SRH at follow-up (2003), logistic regression analyses were performed with three models including information from both waves. For all regression analyses the data used is unweighted. Model 1 included all SES covariates at baseline (2001). Model 2 included all variables from Model 1 and added SRH in 2001. Model 3 added the four disease categories using "neither" as the reference in addition to all SES covariates and SRH in 2001. The latter approach has been validated to examine changes in outcomes using two-panel studies. ${ }^{20}$

To analyze the transitions in SRH between baseline and follow-up, estimated probabilities of SRH at followup were calculated using Model 3. The estimated regression coefficients were used to calculate probability of: 1) reporting poor SRH in 2003 conditional on reporting poor SRH in 2001 ("remain poor"); 2) non-poor SRH in 2003 conditional on poor SRH in 2001 ("improve"); 3) poor SRH or death in 2003 conditional on non-poor SRH in 2001 ("worsen"); and 4) non-poor SRH in 2003 conditional on non-poor SRH in 2001 ("remain healthy"). As stated above, due to low mortality incidence between baseline and follow-up, death was combined with poor SRH in 2003 and then included in the "worsen" transition category. The probabilities for these transitions (remain poor, improve, worsen, and remain healthy) were obtained according to the four disease categories reported at baseline (2001) keeping all other variables at their mean value. To illustrate the results graphically, probabilities were plotted against age. For all models, testing was two-sided using an alpha of 0.05 . All analyses were performed using STATA software version 10 .

\section{Results}

Table I shows characteristics of the sample by disease category. At baseline, individuals reporting chronic diseases are older (mean age 63.5 \pm 9.7 ) than individuals with neither type of disease (60.5 \pm 9.1 ). Women are more likely to report chronic diseases and both types of disease compared to men. Conversely, more men than women report infectious diseases despite a higher percentage reporting neither type of disease (46.9\%). Widowed individuals report having more chronic diseases, and the highest percentage of both types of disease. Conversely, married individuals self-report to be healthier; they report the highest percentage of neither and the lowest percentage of both. More individuals 
TABLE I

Characteristics of the sample at baseline (200I) Stratified by type Of disease $\left(\mathrm{N}=12207^{\mathrm{A}}\right)$

\begin{tabular}{|c|c|c|c|c|c|}
\hline & $\begin{array}{c}\text { Infectious } \\
\text { Mean (SD) }\end{array}$ & $\begin{array}{c}\text { Chronic } \\
\text { Mean }(S D)^{b}\end{array}$ & $\begin{array}{c}\text { Both } \\
\text { Mean }(S D)^{b}\end{array}$ & $\begin{array}{c}\text { Neither } \\
\text { Mean }(S D)^{b}\end{array}$ & $\mathrm{p}$-value \\
\hline Total Sample Size $\left(n=12207^{\circ}\right)$ & 370 & 6062 & I I/4 & 4661 & \\
\hline Age Mean (SD) & $60.5 \quad(9.1)$ & $63.5 \quad(9.7)$ & $\begin{array}{ll}62.8 & (9.5)\end{array}$ & $61.1 \quad(9.5)$ & * \\
\hline
\end{tabular}

Sex

\begin{tabular}{llccc} 
Male & 3.8 & 42.9 & 6.5 & 46.9 \\
\hline Female & 2.4 & 55.4 & 11.4 & 30.8
\end{tabular}

Marital Status

\begin{tabular}{lllll} 
Single $^{c}$ & 2.9 & 47.7 & 9.9 & 39.5 \\
\hline Married $^{d}$ & 3.2 & 48.4 & 8.5 & 39.9 \\
\hline Widowed & 2.2 & 56.8 & 10.9 & 30.2
\end{tabular}

Location

\begin{tabular}{lllll} 
Urban & 2.9 & 50.6 & 9.4 & 37.0 \\
\hline Rural $^{\mathrm{e}}$ & 3.2 & 47.8 & 8.6 & 40.4
\end{tabular}

Education

\begin{tabular}{lllll} 
Low & 3.1 & 50.8 & 9.9 & 36.2 \\
\hline Moderate & 3.2 & 51.3 & 10.3 & 35.2 \\
\hline High & 2.9 & 47.5 & 7.7 & 41.9
\end{tabular}

Assets (tertiles individual level) ${ }^{\mathrm{f}}$

\begin{tabular}{lllll} 
Low & 3.0 & 50.0 & 9.7 & 37.4 \\
\hline Medium & 3.2 & 49.9 & 9.0 & 37.9 \\
\hline High & 2.9 & 49.1 & 8.7 & 39.3
\end{tabular}

Wealth index (tertiles) ${ }^{g}$

\begin{tabular}{lllll} 
Low & 3.3 & 46.8 & 9.1 & 40.8 \\
\hline Medium & 3.0 & 51.0 & 9.1 & 36.9 \\
\hline High & 1.1 & 54.2 & 9.1 & 35.6
\end{tabular}

$* p<0.001 ; * *<<0.01 ; * * * k<0.05$

Data for this study was collected in rural and urban locations in Mexico by the Instituto Nacional de Estadística y Geografía (INEGI) as part of the Mexican Health and Aging Study (MHAS/ENASEM)

Note: $p$-values indicate ANOVA results using an unweighted model to compare the four categories

a Sample size across variables may vary due to missing values

${ }^{b}$ Weights used when estimating means, standard deviations (SD), and percentages

c Includes single, divorced, or separated

Includes married and cohabitation

${ }^{\text {e }}$ Rural regions have $\leq 100000$ inhabitants

${ }^{f}$ Calculated in Mexican pesos

$g$ Wealth index uses the first factor of principal component analysis (Methods section)

with chronic and with both diseases live in urban areas while more individuals with infectious and neither disease live in rural areas.

Additionally, individuals with higher education, higher assets and wealth, report the lowest percentage of both diseases and the highest percentage of neither type of disease. All differences are statistically significant across the four disease categories $(p<.05)$. In summary, older individuals with low SES (rural residence, low education, low assets and low wealth) have higher 
percentages of infectious diseases, and both types of diseases. Conversely, absence of diseases is more common among those with higher SES but reports of chronic diseases increase for these individuals as well.

Table II shows self-reported health in 2003 by baseline characteristics. "Non-poor" includes all individuals reporting excellent, very good, good and fair health. "Poor" includes all individuals reporting poor health in addition to individuals that died. Individuals in poor health at follow-up tended to be older, women, widowed, to live in urban settings, and to have lower education and lower wealth at baseline compared to those reporting non-poor health. When analyzing the disease categories by SRH, those with both types of disease report the highest percentage of poor SRH followed by those with chronic diseases. Those with neither type of disease report the highest percentage with non-poor SRH. In summary at follow-up those reporting poor health or that died had lower SES, tended to live alone and in urban areas compared to those that reported non-poor health.

To check for the predictive power of the diseases further, we examined deaths between waves $(n=517)$. When examining mortality rates at follow-up by disease in 2001, the highest rates were among individuals with both types of disease $(8.2 \%)$, while individuals with neither disease had the lowest mortality rate (3.8\%). Differences were statistically significant between the four disease categories $(p<.001)$.

Table III reports odds of poor SRH in 2003 from three logistic regression models. Model 1 shows that older age, rural residence and lower education at baseline are associated with higher odds of poor SRH at follow-up $(p<.05)$. Conversely, males, those with higher assets and higher wealth have lower odds of poor SRH $(p<.05)$. Model 2 adds poor SRH in 2001. Age and residence remain significant predictors of poor SRH at follow-up. Similarly, higher assets and being male remain significant predictors decreasing the odds of poor SRH or death at follow-up. Wealth is no longer a significant predictor (Odds Ratio [OR] 0.96, Standard Error [SE] 0.03; $p=0.18$ ). Model 2 also shows that poor $\mathrm{SRH}$ in 2001 is the strongest predictor increasing the odds of poor SRH or death in 2003 (OR 4.75, SE 0.27; $p<.0001$ ). Model 3 adds type of disease. Older age, rural residence, lower education and poor SRH in 2001 remain significant predictors $(p<0.05)$ of poor SRH or death in 2003 after controlling for all covariates in Model 2. Higher assets remains a predictor lowering the odds of poor SRH or death in 2003, while being a male is no longer a significant predictor $(p=0.37)$. The three disease categories significantly increase the odds of poor SRH compared to reporting neither type of disease. The odds

\section{TABLE II \\ Self-Reported health IN 2003 OR DeAth BY CHARACTERISTICS AT BASELINE}

\begin{tabular}{|c|c|c|c|}
\hline Total Sample Size $\left(n=10,999^{\circ}\right)$ & $\begin{array}{c}\text { Non-poor } \\
\text { Mean (SD) } \\
8995\end{array}$ & $\begin{array}{c}\text { Poor }^{b} \\
\text { Mean (SD) } \\
2004\end{array}$ & p-value \\
\hline $\begin{array}{l}\text { Age } \\
\text { Mean (SD) }\end{array}$ & $61.6(9.1)$ & $66.5(10.8)$ & * \\
\hline $\begin{array}{l}\text { Assets (LN at the individual level) } \\
\text { Mean (SD) }\end{array}$ & $10.8(3.3)$ & $10.4(3.4)$ & $*$ \\
\hline
\end{tabular}

\begin{tabular}{lllc} 
& $\%$ c & $\%$ c & -value \\
\hline $\begin{array}{l}\text { Age group } \\
50-59\end{array}$ & & & $*$ \\
\hline $60-69$ & 86.1 & 13.9 & \\
\hline $70-79$ & 78.7 & 21.3 & \\
\hline $80+$ & 76.2 & 23.8 \\
\hline
\end{tabular}

\begin{tabular}{|c|c|c|c|}
\hline Sex & & & $*$ \\
\hline Male & 83.3 & 16.7 & \\
\hline Female & 79.3 & 20.7 & \\
\hline Marital Status & & & $*$ \\
\hline Single ${ }^{e}$ & 82.0 & 18.0 & \\
\hline Married $^{f}$ & 81.3 & 18.7 & \\
\hline Widowed & 79.7 & 20.3 & \\
\hline Locationg & & & $*$ \\
\hline Urban & 77.2 & 22.8 & \\
\hline Rural & 85.9 & 14.1 & \\
\hline Education & & & $*$ \\
\hline Low & 75.0 & 25.0 & \\
\hline Moderate & 81.8 & 18.2 & \\
\hline High & 88.8 & 11.2 & \\
\hline Wealth Index (tertiles) ${ }^{\mathrm{h}}$ & & & * \\
\hline Low & 75.6 & 24.4 & \\
\hline Medium & 84.9 & I5.I & \\
\hline High & 82.0 & 18.0 & \\
\hline Type of Disease & & & $*$ \\
\hline Infectious & 80.0 & 20.0 & \\
\hline Chronic & 76.8 & 23.2 & \\
\hline Both & 65.8 & 34.2 & \\
\hline Neither & 88.7 & 11.3 & \\
\hline
\end{tabular}

$* p<0.001 ; * *<<0.01 ; * * * p<0.05$

Data for this study was collected in rural and urban locations in Mexico by INEGI as part of the MHAS/ENASEM Study

Note: $p$-values indicate Pearson's Chi-Square test results for the categorical variables and t-test for the continuous variable (Assets) included using an unweighted model to compare the two categories

a Sample size across variables may vary due to missing values

${ }^{b}$ Poor includes death between baseline and follow-up

c Weights used when estimating means, standard deviations (SD), and percentages

${ }^{d}$ Calculated in Mexican pesos

e Includes single, divorced, or separated

${ }^{\mathrm{f}}$ Includes married and cohabitation

${ }^{g}$ Rural regions have $\leq 100000$ inhabitants

${ }^{\mathrm{h}}$ Wealth index uses the first factor of principal component analysis (Methods section) 
TABLE III

Odds Ratios from Logistic Regression Models of Reporting Poor Health or death in 2003

\begin{tabular}{|c|c|c|c|c|c|c|}
\hline \multirow[b]{2}{*}{ Variable } & \multicolumn{2}{|c|}{ Model I } & \multicolumn{2}{|c|}{ Model 2} & \multicolumn{2}{|c|}{ Model 3} \\
\hline & Odds ratios & Conf. intervala & Odds ratios & Conf. interval ${ }^{a}$ & Odds ratios & Conf. intervala \\
\hline Age & I.04* & $(1.04 ; 1.05)$ & $1.03 *$ & $(1.03 ; 1.04)$ & $1.03 *$ & $(1.03 ; 1.04)$ \\
\hline Male & $0.8 I^{*}$ & $(0.73 ; 0.89)$ & $0.87^{*}$ & $(0.78 ; 0.96)$ & 0.95 & $(0.85 ; 1.06)$ \\
\hline
\end{tabular}

Marital status (married omitted)

\begin{tabular}{lllllll} 
Unmarried & 1.05 & $(0.91 ; 1.22)$ & 1.03 & $(0.88 ; 1.21)$ & 1.05 & $(0.89 ; 1.23)$ \\
\hline Widowed & 0.97 & $(0.85 ; 1.10)$ & 0.99 & $(0.87 ; 1.14)$ & 0.99 & $(0.86 ; 1.13)$ \\
\hline Rural & $1.25^{b}$ & $(1.13 ; 1.39)$ & $1.22^{*}$ & $(1.09 ; 1.36)$ & $1.27^{*}$ & $(1.13 ; 1.42)$ \\
\hline Log assets $(\mathrm{In})$ & $0.98^{*}$ & $(0.97 ; 0.99)$ & $0.98^{*}$ & $(0.97 ; 1.00)$ & $0.98^{*}$ & $(0.97 ; 1.00)$ \\
\hline Wealth index $^{c}$ & $0.94^{*}$ & $(0.89 ; 0.99)$ & 0.96 & $(0.91 ; 1.02)$ & $0.94^{*}$ & $(0.89 ; 1.00)$
\end{tabular}

Education (6 yrs+ omitted)

\begin{tabular}{|c|c|c|c|c|c|c|}
\hline Less than 3 yrs & $1.78 *$ & $(1.57 ; 2.02)$ & $1.61 *$ & $(1.41 ; 1.84)$ & $1.57^{*}$ & $(1.37 ; 1.80)$ \\
\hline From 3 to $5 \mathrm{yrs}$ & $1.38 *$ & (I.20; I.58) & $1.31 *$ & $(1.13 ; 1.5 I)$ & $1.27^{*}$ & (I.09; I.47) \\
\hline Poor $S R H^{d}$ in $200 I$ & & & $4.76^{*}$ & $(4.25 ; 5.32)$ & $4.05 *$ & $(3.60 ; 4.55)$ \\
\hline
\end{tabular}

Type of disease

\begin{tabular}{lll} 
Infectious & $1.55^{*}$ & $(1.12 ; 2.13)$ \\
\hline Chronic & $1.86 *$ & $(1.65 ; 2.10)$ \\
\hline Both (Infectious and chronic) & $2.63 *$ & $(2.20 ; 3.15)$
\end{tabular}

\begin{tabular}{|c|c|c|c|}
\hline Number of observations & $1 \mathrm{I}, 074$ & 10,720 & $|0,43|$ \\
\hline Pseudo R-Square & 0.06 & 0.12 & 0.13 \\
\hline LR chi2 & 676.00 & | 325.28 & | 454.07 \\
\hline Prob>chi2 & $<.01$ & $<.01$ & $<.01$ \\
\hline Correctly classifiede \% & 61.88 & 66.74 & 68.19 \\
\hline
\end{tabular}

$* p<.05$

Data for this study was collected in rural and urban locations in Mexico by INEGI as part of the MHAS/ENASEM Study

Note: Logistic Regressions using unweighted data

a $95 \%$ confidence intervals

b Rural regions have $\leq 100,000$ inhabitants

c Wealth index uses the first factor of Principal Component Analysis (see methods section)

d $\mathrm{SRH}=$ Self-reported health

e We used a sensitivity and specificity analysis and the classification matrix method to determine the model goodness of fit. The measure indicates that the model correctly classifies a percentage of the time

are highest for persons with both types of diseases (OR 2.63, SE 0.24), followed by those with chronic diseases (OR 1.86; SE 0.12) and finally those with infectious diseases (OR 1.55; SE 0.25).*

Figure 1 presents predicted probabilities of transitions in SRH estimated from Model 3 by type of disease

\footnotetext{
* "Liver or kidney infection" is vague and can be perceived to confound the results from the regression models. Results remain virtually unchanged when "liver or kidney infection" is excluded from the "infectious disease category". The OR for only-infectious or only-chronic diseases increases by 0.02 , and the OR for those with both types of disease decreases by 0.04 in model 3 .
}

and SRH at baseline. The probabilities by age show that individuals with both diseases in 2001 have the highest probability of remaining in poor health or worsening than any other disease category. Additionally, individuals with neither type of disease are the least likely to remain in poor health or worsen and are the most likely to remain healthy and improve between waves. As a final point, individuals with chronic diseases have higher probability of remaining in poor health or worsening compared to individuals with infectious diseases.

Additional analyses (available from authors) illustrate that by residence, individuals in urban setting have a higher probability of remaining poor or worsening compared to those in the rural setting. Those living 


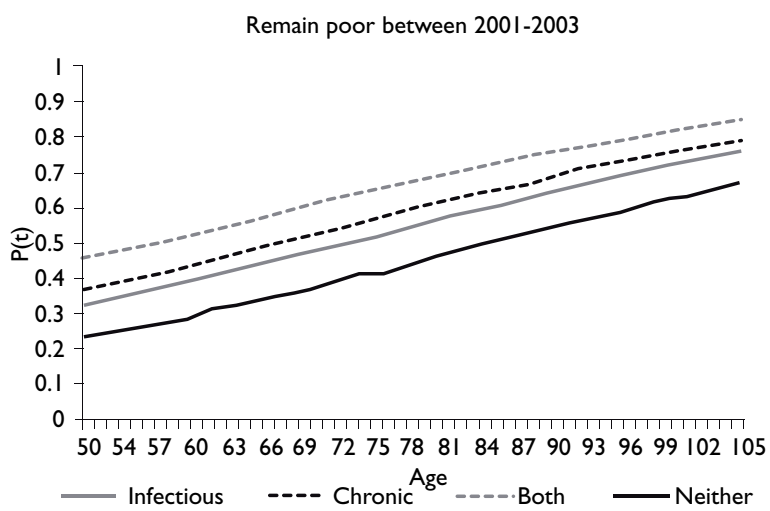

Worsen between 200I-2003

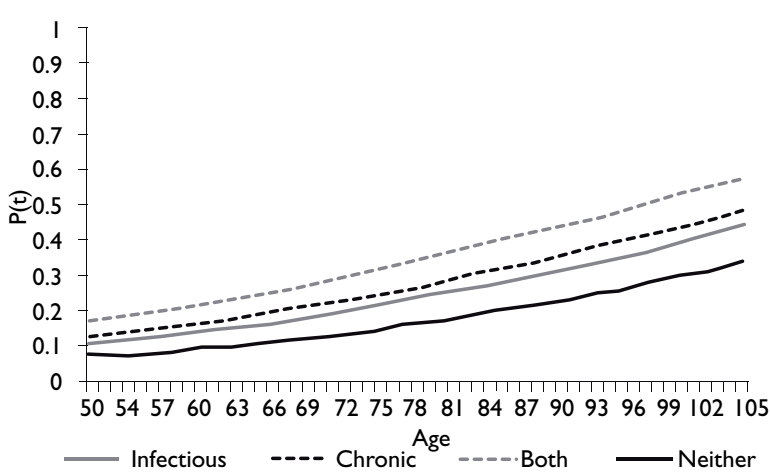

Improve between 200I-2003

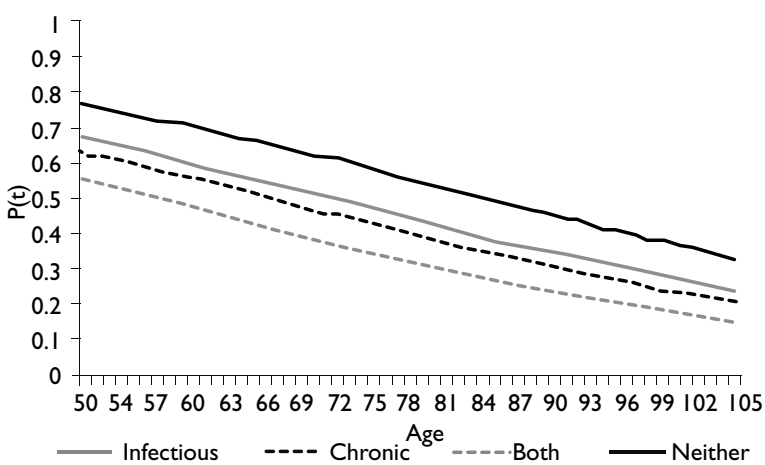

Remain healthy

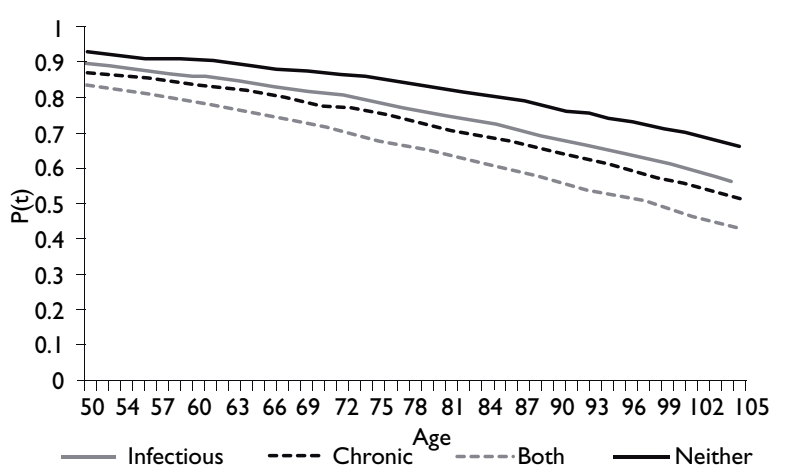

Figure I. Probability of transitions in Self-Reported health between 200 I and 2003 by Age aND type of disease AT BASELINE (200I)

in rural settings have a higher probability of staying healthy and improving. Similarly, those with better SES have higher probability of recovery and remaining healthy and lower probability of worsening or remaining in poor health.

\section{Discussion}

Our study confirms that type of disease affects global health of older adults over time. Individuals with both infectious and chronic diseases have worse SRH compared to those with each type alone. Moreover, individuals with infectious diseases at baseline report better SRH compared to those with chronic diseases or both. In contrast, more individuals with chronic disease or both types of disease at baseline remain with poor $\mathrm{SRH}$ or worsen. Thus, different types of disease increase the odds of poor SRH differently, even after controlling for SES variables.
Our findings contribute to the literature that strives to determine the health dynamics of older adults. ${ }^{21,22}$ Many older adults suffer from chronic conditions that affect their quality of life because of the symptoms they can cause, the limitations imposed by them, and the side effects that treatments originate. In developing countries that are aging rapidly like Mexico, however, many older adults suffer infectious and chronic diseases simultaneously while exposed to poverty, malnutrition and limited healthcare access. ${ }^{23,24}$ Thus, a mixed regime of infectious and chronic diseases poses a unique burden on older adults in Mexico.

For these adults, physiological reserve decreases rapidly and fewer biological resources are available to face acute diseases. ${ }^{25,26}$ This physiologic decline varies noticeably among individuals, manifests differently for each type of disease and is highly correlated with the number and type of alterations an individual suffers. ${ }^{27,28}$ Chronic diseases affect multiple physiologic systems 
simultaneously and cause symptoms once physiologic functioning is disrupted. ${ }^{29}$ On the other hand, infectious diseases are usually limited to a short period of time, and cause symptoms early in their course, affecting function mostly of the immune system. ${ }^{30}$ With these differences in mind, our study attempted to clarify the role different disease categories play on the dynamics of overall health. We conclude that the mixed regime present in countries like Mexico should perhaps be considered as a stage of the transition rather than simply a transition between stages as proposed by researchers using Omran's model. The structural and economic conditions prevalent in developing countries such as Mexico indicate that these countries may spend considerable time in this stage before advancing to a different one.

Our findings also support the concept of deficit accumulation as a predictor of adverse outcomes in older adults. ${ }^{28,31,32}$ In Table III, individuals with both infectious and chronic diseases have the highest risk of reporting poor health and dying. Given that individuals age within a specific context and that this context makes the aging experience unique, analyzing the effect of different contextual variables on health (self-reported in this case) seems useful. This study provides evidence that combinations of different diseases enhances the risk profile for older adults and supports the idea that rather than analyzing diseases as a group, different disease categories provide a more comprehensive approach to understand older adults' health and the aging process, especially in developing countries like Mexico.

Additionally, differences observed between disease categories support the notion that not only the number but also the type of disease determines how the overall health of individuals with different characteristics changes. Infectious diseases have been commonly related to lower SES. Individuals with lower SES are exposed to poorer nutrition, worse sanitary conditions and poor healthcare, increasing their risk for infectious diseases. ${ }^{33}$ Lower SES has also been related to chronic diseases but in a different way. Lower SES is related to higher rates of complications, higher mortality rates and poorer compliance with medical treatment for such diseases. ${ }^{34}$

Our models show that after controlling for type of disease, rural residence and low education are predictors of worsening health. Our study reveals new information related to the epidemiologic transition occurring in Mexico. When two-year transitions in SRH are analyzed, infectious diseases are predictors of recovery while chronic diseases are predictors of worsening SRH, remaining with poor SRH or dying. The importance of this result hinges on the biological differences between infectious and chronic diseases, which appear to have important consequences. Furthermore, these results highlight the value of giving adequate attention to infectious disease eradication despite the exponential rise in chronic diseases in rapidly aging countries like Mexico. Mixed epidemiological regimes like the one in Mexico challenge previous paradigms and must be carefully analyzed in the context of demographic and epidemiologic transitions.

Our study contributes to the literature by examining factors that affect the relationship between SES and $\mathrm{SRH}$. We have identified type of disease as a potential modifier of this relationship that requires further analysis. The mixed regime observed in Mexico suggests that countries may not follow the traditional epidemiologic transition scheme, but rather experience a unique and different stage worth examining. Nevertheless, our study has some limitations. First, all the diseases included in our analyses were self-reported, however, good face validity of self-reports of disease has been found..$^{35}$ Laboratory tests and imaging studies would provide complementary information to better understand the effect of diseases on overall health. Second, grouping the conditions in four categories overlooks several aspects of the disease course for each condition. However, this common categorization provides important information for prevention and public health. Finally, the low rates of mortality limit the conclusions we can draw from our data on the effect of each type of disease and $\mathrm{SRH}$ on mortality incidence. A longer follow-up of the individuals studied will allow for more comprehensive analyses related to mortality as an outcome.

In conclusion, the number and type of disease affect health status and disease progression and modify the relationship between SES and health. The biological mechanism and physiological processes behind each disease are likely to impact how overall health is perceived and reported. Future studies need to consider other SES indicators such as healthcare access and health insurance to determine how health policies can target differences by type of disease. In addition, our study indicates that, despite the epidemiological transition that has heightened the importance of chronic diseases, countries that are aging rapidly like Mexico need to pay closer attention to infectious diseases among older adults, and keep resources focused on their control and eradication. Disregarding the relevance of infectious diseases for public health in favor of chronic conditions may be unjustified and somewhat premature.

\section{Acknowledgements}

This work was supported by the National Institute on Aging at NIH [grant R01 AG025533. Data for this study comes from the Mexican Health and Aging Stu- 
dy (MHAS), also funded by the National Institute on Aging (R01 AG018016). Rafael Samper-Ternent is also supported in part by the Health Resources and Services Administration, the National Cancer Institute, the National Center for Research Resources and the National Institute on Disability and Rehabilitation Research [grants UB4HP19213-01, R01 CA133069-01, UL1RR029876). Alberto Palloni's research is supported by other grants from the National Institute of Aging, the Fogarty International Center and the Robert Wood Johnson Investigator Awards in Health Policy [grants R01 AG016209, R37 AG025216, 5D43TW001586 and Research Grant ID \# 67212]. Research at the Center for Demography and Ecology (CDE) and the Center for Demography of Health and Aging (CDHA), University of Wisconsin-Madison is supported by the NICHD Center Grant 5R24HD04783 and by NIA Center Grant 5P30AG017266. Infrastructure support provided by the Sealy Center on Aging at the University of Texas Medical Branch.

Declaration of conflict of interests. The authors declare that they have no conflict of interests.

\section{References}

I. Omran AR. The epidemiologic transition. A theory of the epidemiology of population change. Milbank Mem Fund Q 1971;49:509-538.

2. Chen F, Yang Y, Liu G. Social Change and Socioeconomic Disparities in Health over the Life Course in China: A Cohort Analysis. Am Sociol Rev, 2010;75: $126-150$.

3. Riley JC, Alter G. The epidemiologic transition and morbidity. Ann Demogr Hist (Paris) 1989:199-213.

4. Wilmoth JR, Horiuchi S. Rectangularization revisited: variability of age at death within human populations. Demography 1999;36: 475-495.

5. Gaziano JM. Fifth phase of the epidemiologic transition: the age of obesity and inactivity. JAMA 2010;303:275-276.

6. Cubillos-Garzon LA, Casas JP, Morillo CA, Bautista LE. Congestive heart failure in Latin America: the next epidemic. Am Heart J 2004; 147:4|2-4I7.

7. Palloni A, Pinto-Aguirre G, Pelaez M. Demographic and health conditions of ageing in Latin America and the Caribbean. Int J Epidemiol 2002;31:762-77I.

8. Arredondo A. Costs and financial consequences of the changing epidemiological profile in Mexico. Health Policy 1997;42:39-48.

9. Chandola T, Jenkinson C. Validating self-rated health in different ethnic groups. Ethn Health 2000;5:15I-159.

I0. Idler EL, KasI SV. Self-ratings of health: do they also predict change in functional ability? J Gerontol B Psychol Sci Soc Sci 1995;50:S344-S353. II. Mexican Health and Aging Study (2009). [Electronic version]. [Retrieved: 8-|2-20II]. Available from: www.mhas.pop.upenn.edu

12. Wong R, Espinoza M, Palloni A. Mexican older adults with a wide socioeconomic perspective: health and aging. Salud Publica Mex 2007;49 Suppl 4: S436-S447.

13. McGee DL, Liao Y, Cao G, Cooper RS. Self-reported health status and mortality in a multiethnic US cohort. Am J Epidemiol I999; 149, 4I-46.
14. Wong R, Pelaez M, Palloni A. [Self-reported general health in older adults in Latin America and the Caribbean: Usefulness of the indicator]. Rev Panam Salud Publica 2005; 17:323-332.

15. Ciftci EE, Bago d'Uva T, van Doorslaer E, van Lenthe F]. Self-percieved Health and Longevity: Do Dynamics Matter? (2009). [Retrieved: 8-I2-20I I]. Available from: http://www.york.ac.uk/res/herc/documents/ wp/09_31.pdf

16. Wong R, Espinoza M. Imputation of Non-Response on Economic Variables in the Mexican Health and Aging Study. MHAS/ENSAME 2003. 17. O'Donell O, van Doorslaer E, Wagstaff A, Lindelow M. Measurement of Living Standards. In: Analyzing Health Equity Using Household Survey Data: A Guide to Techniques and Their Implementation. 2008: The World Bank: 69-82.

18. Wong R, Espinoza-Higgins M. Income and assets of the population of middle and old age in Mexico. Papeles de Población 2003;37:129-166. 19. Filmer D, Pritchett LH. Estimating wealth effects without expenditure data - Or tears: An application to educational enrollments in states of India. Demography 2001;38:115-132.

20. Johnson D. Two-wave Panel Analysis: Comparing Statistical Methods for Studying the Effects of Transitions. J Marriage Fam 2005;67:1061-1075. 21. Cott CA, Gignac MA, Badley EM. Determinants of self rated health for Canadians with chronic disease and disability. J Epid Comm Health 1999;53:731-736.

22. Mathers CD, Vos ET, Stevenson CE, Begg SJ. The burden of disease and injury in Australia. Bull World Health Organ 200I;79:1076-1084. 23. Gonzalez CA, Ham-Chande R. Funcionalidad y salud: una tipología del envejecimiento en México [Functional status and health: a typology of aging in Mexico]. Salud Publica Mex 2007;49:S448-S458.

24. Kuri-Morales P, Emberson J, Alegre-Diaz J, Tapia-Conyer R, Collins R, Peto $R$, et al. The prevalence of chronic diseases and major disease risk factors at different ages among 150,000 men and women living in Mexico City: cross-sectional analyses of a prospective study. BMC Public Health 2009;9:9.

25. Sawhney R, Sehl M, Naeim A. Physiologic aspects of aging: impact on cancer management and decision making. Part I. Cancer J 2005; I :449-460. 26. Sehl M, Sawhney R, Naeim A. Physiologic aspects of aging: impact on cancer management and decision making. Part II. Cancer J 2005; | I:46 I-473. 27. Lipsitz LA. Physiological complexity, aging, and the path to frailty. Sci Aging Knowledge Environ 2004; 16.

28. Rockwood K, Mitnitski A. Frailty in relation to the accumulation of deficits. J Gerontol A Biol Sci Med Sci 2007;62, 722-727.

29. Ben-Shlomo Y, Kuh D. A life course approach to chronic disease epidemiology: conceptual models, empirical challenges and interdisciplinary perspectives. Int J Epidemiol 2002;31:285-293.

30. Hall AJ, Yee LJ, Thomas SL. Life course epidemiology and infectious diseases. Int J Epidemiol 2002;31:300-30I.

3I. Andrew MK, Mitnitski AB, Rockwood K. Social vulnerability, frailty and mortality in elderly people. PLoS One 2008;3:e2232.

32. Song X, Mitnitski A, Rockwood K. Prevalence and 10-year outcomes of frailty in older adults in relation to deficit accumulation. J Am Geriatr Soc 2010;58:68I-687.

33. House JS, Kessler RC, Herzog AR. Age, socioeconomic status, and health. Milbank Quarterly 1990;68:383-4I I.

34. Feinstein JS. The relationship between socioeconomic status and health: A review of the literature. Milbank Quarterly 1993;71:279-322. 35. Okura Y, Urban LH, Mahoney DW, Jacobsen SJ, Rodeheffer RJ. Agreement between self-report questionnaires and medical record data was substantial for diabetes, hypertension, myocardial infarction and stroke but not for heart failure. J Clin Epidemiol 2004;57:1096-I I03. 